greater appreciation of life contributes to this. Again, the value of the individual was stressed and it was recommended that the Federation should use its influence to make mass media such as films and radio less sensational and more inspiring to the individual. Cheap means of printing and the suppression of overnationalistic text-books would be helpful, too, as would schemes for the exchange of students and teachers between rural schools and others. It was further recommended that the value of the humanities in general education should be emphasized, while recognizing the importance of scientific and technological factors.

The group which discussed the ethical basis of human values came to the conclusion that the essential value at which man ought to aim is the increasing development of those powers of soul and spirit which contribute to build up a harmonious personality and society. Special efforts should be made to counteract the levelling tendencies of technology by stressing the necessity of individual decision.

In studying the organization of work, a further group considered it should be so arranged as to protect the personality of the individual. Suggested practical means to this end were: vocational guidance for all, and an effort to find ways of overcoming the effects of monotonous work.

The fifth group, in dealing with the impact of modern techniques on non-industrial communities, reached the conclusion that the greatest precaution should be used in the introduction of foreign ways and standards of living into non-industrial countries. Accepting the fact that technology's greatest contribution lies in the service of others, this group stressed that this service can be performed on the spiritual plane as well as on the material.

In summing up the findings of the groups, Dr. Henrici said that in this time of transition and crisis the threat to human values comes not only from external nature but also from civilization itself. It is the task of educated people everywhere to seek the truth with fervour and to follow it with courage. It is a more difficult task than to live according to the precepts of the loud-speaker. The power of decision lies with each individual and therefore with the human race. The individual is not himself responsible for the decisions taken by the race, but in living his daily life he can influence them for good or ill.

\section{PARTICLES OF COSMIC RADIATION}

A

$\mathrm{N}$ international conference on cosmic radiation, organized by the University of Toulouse and under the patronage of the International Union of Pure and Applied Physics, was held at Bagnères de Bigorre during July 6-12. The president of the conference was Prof. P. M. S. Blackett, and the secretary-general Prof. L. Leprince-Ringuet. In opening the conference Prof. Blackett said that it had been decided to restrict the discussion in the main sessions to the narrow but important field of $V$-particles and heavy mesons. It was hoped thus to make an effective contribution to a subject of great contemporary interest for the development of our knowledge of elementary particles. This aim was reflected in the attendance at the conference, and among the two hundred members were representatives of many of the laboratories actively engaged in the subject.

The main sessions of the conference were divided into two groups. In the first seven, the experimental facts were described and discussed; in the last three, the whole body of evidence was reviewed, and an estimate made of the present state of knowledge. In describing the most important results and conclusions, it will be convenient first to consider particles more massive than the neutron; and secondly, those intermediate in mass between the $\pi$-meson and the proton.

\section{Particles Heavier than the Proton}

It is now established that the heavy neutral $V$-particles discovered by Rochester and Butler $\left(V_{1}{ }^{\circ}\right)$ decay into a proton and a negative $\pi$-meson with an energy release of about $37 \mathrm{MeV}$. :

$$
V_{1}{ }^{0} \rightarrow P+\pi^{-} ; Q=37 \pm 3 \mathrm{Mer} .
$$

The mean lifetime of the particles is $\sim 3 \cdot 3 \pm 1 \cdot 0 \times$ $10^{-10}$ sec. There is good evidence that they can be produced in nuclear interactions in which the available kinetic energy is insufficient to provide all their rest-mass. They are therefore believed to result from the transformation of nucleons, and, in this sense and since they decay into protons, they may be termed 'excited nucleons'. An outstanding problem of great interest is whether the particles are commonly produced singly or in pairs, a question which it may be possible to resolve by determining the minimum energy required for their production in the collision of protons and $\pi$-mesons with nuclei.

There is now strong evidence from work with photographic plates for the existence of charged particles more massive than the proton, each of which decays into a neutral particle and a $\pi$-particle according to the equation :

$$
J \pm \rightarrow N+\pi \pm .
$$

If the secondary neutral particle is a neutron, the release of energy, $Q$, is $\sim 130 \mathrm{MeV}$., and the mass of the parent particle, $m_{J}, \sim 2,360 m_{\ell}$. Five examples have now been observed in photographic emulsions, in three of which the primary particles decay in flight. The mean lifetime appears to be of the order $4 \times 10^{-11}$ sec. There is tentative evidence for an alternative mode of decay $J+\rightarrow P \pm+\pi^{0}$, but this remains to be confirmed.

There is good evidence from experiments with Wilson chambers, but also not decisive, for a massive charged particle which decays into a negative $\pi$-meson and a neutral $V_{2}{ }^{0}$-particle according to the equation $\Lambda^{-} \rightarrow V_{1}^{0}+\pi^{-}$. In addition to the original event, which can be interpreted in this way, found by Armenteros et al., three similar examples have been observed by Leighton and his colleagues. It is possible that the particles $J$ and $\Lambda$ are identical, in which case their mass is $\sim 2,700 m_{6}$.

Further examples have now been observed of the process, first observed by Danysz, in which a heavy nuclear fragment ejected from a nuclear explosion reaches the end of its range and disintegrates. It appears that $\pi$-mesons are frequently emitted as one of the products of the secondary disintegration. It is possible that these events are due to the presence, in the nuclear fragment, of a nucleon in an excited state; but alternative explanations cannot at present be excluded. 


\section{Heavy Mesons}

Of the heavy charged mesons with masses in the interval $900-1,500 m_{e}$, it is established that particles of mass $970 \pm 5 m_{e}$ exist which decay into three $\pi$-mesons. The particles are referred to as $\tau$-mesons; $\tau \rightarrow 3 \pi$. Seventeen examples have been recorded in photographic plates, in all of which the particles decay 'at rest'; and of the fifty-one secondary $\pi$-particles from these events, eleven have been observed to reach the end of their range. Eight of them have thus been identified as positive, and three as negative, $\pi$-particles. This fact, and the observed co-planarity of the directions of emission of the three $\pi$-particles from each decaying $\tau$-meson, make it probable that all, or most, of the particles which decay in this mode when arrested in photographic emulsions are positively charged. A few examples of the decay 'in flight' of $\tau$-mesons have been observed in Wilson chambers. In one case, the parent particle was negatively charged. The $\tau$-mesons observed in cloud-chambers occur infrequently compared with charged $V$-particles, $N_{\tau} \pm / N_{V \pm}$ being $\sim 1 / 20$.

It is established that some heavy mesons, when arrested in photographic emulsions or in metal plates, decay with the emission of $\mu$-mesons. This follows, on one hand, from the direct observation of the $\beta$-decay of the secondary mesons, or from massmeasurements in photographic plates; and, on the other hand, from experiments with expansion chambers in which the secondary particles are observed to traverse a total distance equal to several "interaction-lengths' in metal plates, without giving decisive evidence for a nuclear interaction. The energy of the secondary $\mu$-meson varies widely-from very low values up to at least $200 \mathrm{MeV}$. It is therefore necessary to assume that the $\mu$-meson is accompanied by at least two neutral particles. Particles decaying in this mode are referred to as $x$-mesons ; $x \rightarrow \mu+2$ neutral particles.

Experiments with photographic plates suggest that heavy mesons may sometimes decay with the emission of a $\pi$-meson of energy $\sim 110 \mathrm{MeV}$. The evidence is not decisive ; it receives no support from observations with cloud chambers, but the latter are not inconsistent with the view that heavy mesons sometimes decay in this mode. Since the energy of the secondary $\pi$-mesons appears to be unique, the transformation is written, tentatively: $\chi \rightarrow \pi+1$ neutral particle.

Experiments with Wilson chambers indicate that photons, with energy 100-200 MeV., are sometimes produced in the decay of heavy particles. The photons, detected by the small cascades of electrons to which they give rise, appear to recoil from the secondary charged particle. The observations are compatible with a decay of the parent into two particles only, a charged particle and a photon, but this interpretation does not necessarily follow from the observations and must be treated with reserve.

All the direct measurements, made hitherto, of the mass of heavy charged mesons which come to rest and decay into a single charged particle are consistent with the view that all the particles have masses near, or equal to, that of the $\tau$-meson. This result, if taken alone, makes it permissible to assume, tentatively, that the particles distinguished phenomenologically as $\tau-, \quad x$ - and $\chi$-mesons represent alternative modes of decay of particles of a single type, but it does not prove this assumption to be true.

There are two pieces of experimental evidence which suggest that among the heavy mesons are some which have masses significantly greater than that of the $\tau$-meson. The first piece of evidence is the observation of the maximum energy of the $\mu$-mesons produced in the decay of $x$-mesons. If the $\tau$-meson has a mass of $970 m_{e}$, the maximum energy of the $\mu$-mesons, set by the conservation laws and assuming both neutral particles to have negligible rest-mass, is $\sim 150 \mathrm{MeV}$.; the corresponding values of momentum, $p$ and $p \beta$, are $\sim 233 \mathrm{MeV} . / c$ and $\sim 211 \mathrm{MeV} . / c$, respectively. Observations by expansion chamber give no evidence for secondary particles with energies greater than this limit. On the other hand, measurements with plates provide several examples in which the secondary particles appear to have values of $p \beta$ substantially greater than that corresponding to the limit. It is well known that determinations of $p \beta$ by the scattering method can give high values due to statistical fluctuations. The probability that the high values in question are to be so interpreted appears, however, to be small--less than 1 per cent. To bring these observations into accord with the assumptions that $\tau \equiv x$ or that $m_{\tau} \sim m_{\boldsymbol{x}}$, it is necessary to assume either that there are unsuspected sources of error in the measurements, or that there are alternative physical processes involved. It is possible, for example, that some of the heavy mesons observed to decay are negatively charged. If weakly interacting, they might then decay while moving with high velocity in bound orbits around nuclei; and if so, the energy spectrum of the secondary particles might be greatly modified as compared with that of the positive particles. Such an effect is believed to exist in the case of negative $\mu$-mesons captured by nuclei of low atomic number.

The second piece of evidence is that measurements by the photographic method of the masses of heavy mesons directly emitted with great velocity $(\beta \sim 0.7)$ from nuclear disintegrations give a mean value of about $1,210 \mathrm{me}_{e}$; and there are no individual values in the mass spectrum less than $950 m_{e}$. It is reasonable to assume tentatively that the fast heavy mesons created in the high-energy events are identical with those with approximately the same velocity, observed in experiments with Wilson chamber, which appear to have masses of the order of $920 \pm 40 \mathrm{~m}_{6}$. The later values are not significantly different from those of the slow particles already discussed.

The above apparent contradiction in the mass values can be resolved, and the assumptions that $x \equiv \tau$, or that $m_{\varkappa} \sim m_{\tau}$, can be maintained, if it is assumed that the mass measurements by the photographic method are subject to unrecognized systematic errors. Alternatively, it may be assumed that the heavy particles of mass $\sim 1,210 m_{b}$ are indeed emitted from high-energy disintegrations, but that they decay in flight, with a mean lifetime of the order of $3 \times 10^{-11}$ sec., and transform into particles of mass $\sim 970 m_{\ell}$. In general, such a transformation should lead to a deviation in the track of the particle, but the deviations may be small if most of the difference in mass appears as the restmass of the secondary neutral particle or particles.

\section{Nuclear Capture of Negative Heavy Mesons}

There appears to be a large disparity between the number of heavy mesons observed to decay in nuclear emulsions and the number which interact with nuclei. The evidence obtained with Wilson chambers shows that positively and negatively charged $V$-particles occur with approximately equal fre- 
quency. Since the particles appear to be directly created in nuclear interactions, it would be reasonable to assume that they are strongly interacting. If so, when arrested in solid substances, they would be expected to produce observable disintegrations. Very few such disintegrations have been found, although many laboratories have made a well-directed search.

It is difficult, at present, to make a reliable estimate of the ratio of the number of heavy mesons decaying to the number producing a recognized nuclear interaction because of the different mothods of search employed in different laboratories, but a conservative estimate suggests that it is at least five to one. If the particles observed to decay are all positive, it then follows that only about a fifth of the negative heavy mesons, when arrested in photographic emulsions, produce effects due to nuclear capture comparable with those due to negative $\pi$-mesons. Alternatively, some of the particles may be weakly interacting so that, as with $\mu$-mesons, some of them, even when negative, may decay.

\section{Neutral Heavy Mesons}

of the neutral particles less massive than the proton, it is now established that there is one type which decays into two charged mesons, one of which is a $\pi$-particle, and probably both: $\theta^{0} \rightarrow \pi^{+}+\pi^{-}$; $Q=214 \mathrm{MeV}$. The mean lifetime of these particles is $\sim 10^{-10}$ sec. If the secondary charged particles are both $\pi$-mesons, the mass of the parent particle $m_{\theta^{*}}=971 \pm 10 m_{\epsilon}$, a value equal to that of the $\tau$-meson within the limits of experimental error.

There is evidence for other types of heavy neutral particles, referred to as $V_{3}{ }^{0}$, which appear to decay into a heavy meson and a light meson $(\pi$ or $\mu$ ), but it remains to be confirmed.

The conference gave an impressive demonstration of the great profit to be derived from meetings between representatives of different scientific traditions. The presence of many experts in the various experimental methods ensured a most informed and critical approach to the important questions which were reviewed, and the conferenco was memorable for the very serious level of the discussions, and the cordial relations between its members.

C. F. Poweli

\section{PROGRAMME OF AUSTRALIAN ANTARCTIC EXPLORATION}

\section{By Sir DOUGLAS MAWSON, O.B.E., F.R.S.}

D

URING the past six years, the Commonwealth Government of Australia has, through its organization known as the Australian National Antarctic Research Expedition, prosecuted scientific investigations in the Far South. As long ago as 1939 tentative plans were formulated to continue with the scientific exploration of Antarctica. At that time it was proposed to organize such work under the rgis of the Australian universities, with the expectation of maintaining one or more shore research stations on the Antarctic mainland, there to be serviced from Australia by employment of the Wyatt Earp, which in 1939 the American explorer Lincoln Ellsworth had disposed of to the Commonwealth Government. However, the advent of war postponed such proposals and the Wyatt Earp was placed under the care of the Royal Australian Navy.
At the termination of hostilities the matter was again pressed, representations to the Government being made by the National Research Council.

Eventually, early in 1947, the Government agreed to meet the cost of refitting the Wyatt Earp as required for explorations in Antarctica and to establish and conduct research stations in the subantarctic on both Macquarie Island and Heard Island : the former about 900 miles S.S.E. of Hobart, the latter about 2,500 miles S.W. of Perth. GroupCaptain Stuart Campbell, who had had Antaretic experience as chief aviator of the B.A.N.Z.A.R. Expedition of 1929-31, was seconded to be chief executive officer in command of the undertaking. Much was accomplished in that first year. On Macquarie Island and on Heard Island well-equipped stations were established and operated during the year 1948. Also in the summer of 1947-48, with Campbell on board and a staff of several scientists, including Mr. P. G. Law, the Wyatt Earp, manned by a naval crew under the command of Commander Karl Oom, made a short, late-season cruise into the pack-ice, including a visit to the Balleney Islands.

The report furnished at the conclusion of the cruise indicated that the Wyatt Earp was too small for the undertakings planned, her capacity being barely sufficient for the naval crew, leaving insufficient accommodation for the transport of shore-station staff and their equipment. The antaretic section of the programme was then suspended until a suitable vessel of greater capacity should be made available. The sub-antarctic stations, which were at that time being serviced annually by a naval L.S.T. vessel, were in the meantime to continue in operation.

Group-Captain Campbell returned to his post-war assignment in Commonwealth Civil Aviation Controls, and for several years since has been on duty in Siam as Commonwealth officer on Ioan to help reorganize civil aviation in that country.

In the meantime, the Department of External Affairs established an Antarctic Division to take care of Australian activities in the Far South. Mr. P. G. Law is the officer in charge.

The stations at Macquarie Island and at Heard Island have continued to function without a break, the personnel being relieved each year by the transport of new staff and supplies. The meteorological programme has been specially stressed, since it is hoped that by a detailed study of the movements of air masses between Antarctica and Australia the possibility of forecasting the weather of southern Australia may be enhanced.

Considerable attention is being given to geophysics, radiophysies and cosmic ray studies, and there is ample provision for cartographical, biological and geological activities. Already there has been amassed a very substantial and valuable addition to knowledge of the sub-antarctic in the Australian region.

What is now due to be undertaken is the extension of this programme to the antarctic mainland. This depends upon the provision of adequate transport. The design of a vessel especially to meet the needs of exploration within the pack-ice zone was submitted through the Navy Department, but, on account of cost, has been shelved. Nevertheless, provision has now been made for the establishment next summer of a base station on the antarctic mainland. Final approval and an outline of plans was recently notified by the Right Hon. R. G. Casey, Minister of External Affairs. 